\title{
Nuclear Quantum Effects in Hydrophobic Nanoconfinement
}

\author{
Buddha Ratna Shrestha, ${ }^{\dagger, \|}$ Sreekiran Pillai, ${ }^{\dagger, \|}$ Adriano Santana, ${ }^{\dagger}$ Stephen H. Donaldson Jr., ${ }^{\ddagger}$
}

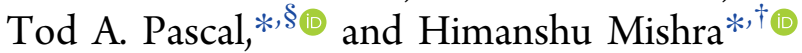

${ }^{\dagger}$ King Abdullah University of Science and Technology (KAUST), Water Desalination and Reuse Center (WDRC), Biological and Environmental Sciences and Engineering (BESE) Division, Thuwal 23955-6900, Saudi Arabia

${ }^{\ddagger}$ Département de Physique, Ecole Normale Supérieure/PSL Research University, CNRS, 24 rue Lhomond, 75005 Paris, France

${ }^{\S}$ ATLaS Laboratory, Department of NanoEngineering and Chemical Engineering, University of California, San Diego, La Jolla, California 92023, United States

\section{Supporting Information}

ABSTRACT: Nuclear quantum effects (NQEs) in water arise due to delocalization, zero-point energy (ZPE), and quantum tunneling of protons. Whereas quantum tunneling is significant only at low temperatures, proton delocalization and $\mathrm{ZPE}$ influence the properties of water at normal temperature and pressure (NTP), giving rise to isotope effects. However, the consequences of NQEs for interfaces of water with hydrophobic media, such as perfluorocarbons, have remained largely unexplored. Here, we reveal the existence and signature of NQEs modulating hydrophobic surface forces at NTP. Our experiments demonstrate that the attractive hydrophobic forces between molecularly smooth

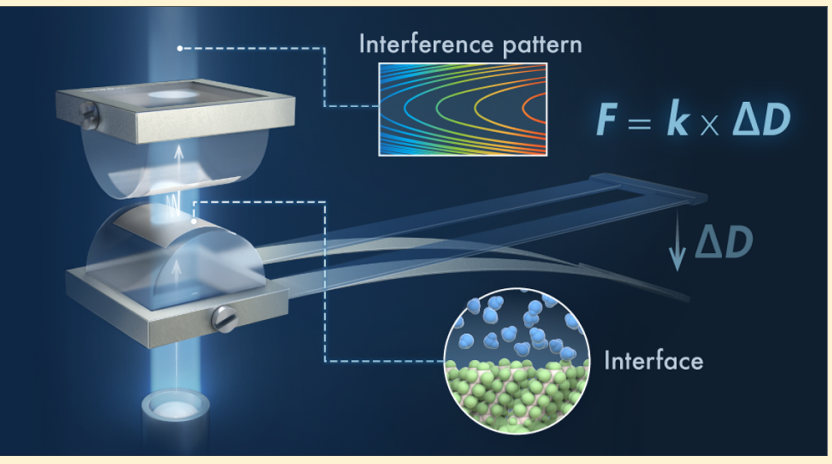
and rigid perfluorinated surfaces in nanoconfinement are $\approx 10 \%$ higher in $\mathrm{H}_{2} \mathrm{O}$ than in $\mathrm{D}_{2} \mathrm{O}$, even though the contact angles of $\mathrm{H}_{2} \mathrm{O}$ and $\mathrm{D}_{2} \mathrm{O}$ on these surfaces are indistinguishable. Our molecular dynamics simulations show that the underlying cause of the difference includes the destabilizing effect of ZPE on the librational motions of interfacial $\mathrm{H}_{2} \mathrm{O}$, which experiences larger quantum effects than $\mathrm{D}_{2} \mathrm{O}$.

\begin{abstract}
A lthough progressively less influential with increasing temperatures, nuclear quantum effects (NQEs) are known to affect the static and dynamic properties of bulk water $^{1-4}$ as well as the water-mediated interactions between small hydrocarbons and perfluorocarbons. ${ }^{5}$ Whereas the bulk properties of $\mathrm{H}_{2} \mathrm{O}$ and $\mathrm{D}_{2} \mathrm{O}$ liquids at NTP, including the surface tension, refractive index, dielectric constant, and density, are quite $\operatorname{similar}^{3,6}$ (Table S1a), their infrared (IR) vibrational spectra differ significantly; notably, the $\mathrm{O}-\mathrm{D}$ stretch in $\mathrm{D}_{2} \mathrm{O}$ is much more localized than the $\mathrm{O}-\mathrm{H}$ stretch (Table S1a). ${ }^{6-8}$ Infrared attenuated total reflection spectra of $\mathrm{D}_{2} \mathrm{O}$ show three distinct peaks at $2395 \mathrm{~cm}^{-1}, 2479 \mathrm{~cm}^{-1}$, and $2587 \mathrm{~cm}^{-1}$, whereas $\mathrm{H}_{2} \mathrm{O}$ spectra show a broad band in the same region that indicates much more delocalized and excitonic vibrational modes. ${ }^{7}$ We are interested in exploring the role of NQEs in hydrophobic interactions that are defined as mutually attractive forces experienced by apolar solutes ${ }^{9,10}$ and surfaces ${ }^{11-14}$ in water. In this context, researchers have compared hydrophobic transfer energies, ${ }^{15,16}$ rates of chemical reactions in $\mathrm{H}_{2} \mathrm{O}$ and $\mathrm{D}_{2} \mathrm{O},{ }^{15,17,18}$ and chaotropic effects of ions, ${ }^{19}$ but a direct comparison of hydrophobic surface forces in $\mathrm{H}_{2} \mathrm{O}$ and $\mathrm{D}_{2} \mathrm{O}$ has not been reported. The resulting insights into NQEs influencing hydrophobic surface forces in nanoconfinement can be useful in understanding a diverse array of nanoscale phenomena in natural and applied contexts, such as
\end{abstract}

in nanofluidics, ${ }^{20,21}$ self-assembly, ${ }^{22-24}$ and cellular processes, ${ }^{5,24,25}$ and might aid in the rational design of nanoscale devices and separation processes. Here, we present evidence of NQEs modulating hydrophobic surface forces in nanoconfinement through complementary experiments and molecular simulations.

To examine hydrophobic surface forces, we used molecularly smooth muscovite mica films $(\approx 5 \mu \mathrm{m}$ thick, with a $50 \mathrm{~nm}$ thick silver layer on the backside) glued onto cylindrical and transparent silica discs with a radius of curvature $(R)$ of $\approx 2 \mathrm{~cm}$ (Figure 1a and Supporting Information section I.1). Freshly cleaved mica surfaces are hydrophilic, characterized by intrinsic contact angles, $\theta_{0}<5^{\circ}$. To achieve smooth and robust hydrophobic surfaces for our experiments, we developed a technique for covalently grafting perfluorodecyltrichlorosilane (FDTS) molecules onto mica [Supporting Information section I.2 and Figures S1-S4, with atomic force microscopy (AFM) and transmission electron microscopy (TEM) characterizations in Figures S2 and S3, respectively]. The resulting surfaces were hydrophobic, characterized by intrinsic contact angles $\left(\theta_{\mathrm{o}}=112 \pm 1^{\circ}\right)$, advancing angles $\left(\theta_{\mathrm{A}}=118 \pm 2^{\circ}\right)$, and

Received: June 25, 2019

Accepted: July 31, 2019

Published: July 31, 2019 

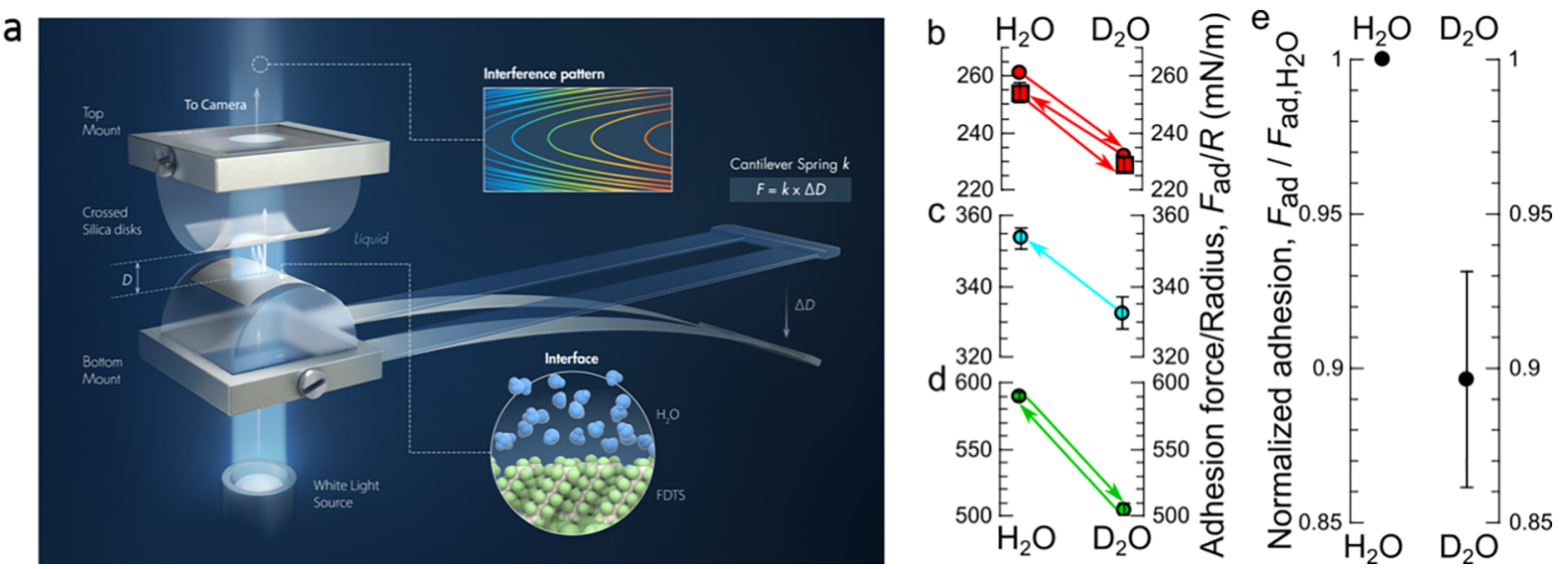

Figure 1. (a) Schematic of our surface force apparatus (SFA). The insets show a schematic representation of the FDTS-water interface and a representative optical interference pattern. (b and c) Representative hydrophobic forces measured between FDTS-coated mica surfaces measured in $\mathrm{H}_{2} \mathrm{O}$ and $\mathrm{D}_{2} \mathrm{O}$ using the SFA. (d) Representative hydrophobic forces measured between FDTS- and ODT-coated surfaces. (e) The data demonstrate that the hydrophobic adhesion between two FDTS-coated mica surfaces is almost $10 \%$ higher in $\mathrm{H}_{2} \mathrm{O}$ than in $\mathrm{D}_{2} \mathrm{O}$. The uncertainties (standard deviation of $1 \sigma$ ) are indicated by the error bars. Figure 1a was produced by Xavier Pita, Senior Scientific Illustrator at King Abdullah University of Science and Technology (KAUST).

receding angles $\left(\theta_{\mathrm{R}}=100 \pm 2^{\circ}\right)$ for the $\mathrm{H}_{2} \mathrm{O} /$ air and $\mathrm{D}_{2} \mathrm{O} /$ air systems (Figure $\mathrm{S} 4$ and Table $\mathrm{S} 1 \mathrm{~b}$ ). We found no significant differences in the wetting behaviors of $\mathrm{H}_{2} \mathrm{O}$ and $\mathrm{D}_{2} \mathrm{O}$ drops on FDTS/mica surfaces. The water repellence of coatings can vary with time due to surface degradation/deformation ${ }^{26}$ and contamination. ${ }^{27}$ We therefore investigated the stability of our FDTS/mica surfaces immersed in water for periods equal to the duration of our surface force measurements, and we found that they remained stable for at least $6 \mathrm{~h}$ (Table S1b).

Next, we quantified the forces between our FDTS surfaces in water at angstrom-scale resolution using a surface force apparatus (SFA) ${ }^{28}$ Silica discs, with FDTS/mica/silver films glued onto them, were placed in a cross-cylinder geometry, such that the back-silvered layers facilitated the accurate determination of the distances between the surfaces by white light multiple-beam interferometry ${ }^{28}$ (Figure 1a, Methods). To measure the forces, we brought the surfaces together at slow speeds of $\sim 10 \mathrm{~nm} \mathrm{~s}^{-1}$ (Supporting Information section I.5) immersed in $5 \mathrm{mM} \mathrm{KCl}$ degassed solutions of $\mathrm{H}_{2} \mathrm{O}$ or $\mathrm{D}_{2} \mathrm{O}$. We found that the FDTS surfaces attracted each other due to hydrophobic forces starting at distances $(D)$ of $\sim 10 \mathrm{~nm}$, followed by a jump-in instability bringing the surfaces into contact (typical areas of $\approx 100 \mu \mathrm{m}^{2}$ ), as reported by others. ${ }^{11,13,29}$ The total adhesion force, $F_{\mathrm{ad}}$, was measured by separating the surfaces from contact, upon which a spring instability resulted in a jump-out from contact (Figure 1a, Methods, and Figure S5). From the precise values of the jumpout distance, $D_{\text {jump }}$, and the spring constant, $k$, we calculated the total adhesion force with the equation $F_{\text {ad }}=k \times D_{\text {jump }}$.

We measured the adhesion in $\mathrm{H}_{2} \mathrm{O}$ and then in $\mathrm{D}_{2} \mathrm{O}$ and observed a significant decrease upon changing the solution (Figure 1b). To confirm that this result is not due to surface contamination or degradation, each of which can strongly affect measurements of hydrophobic interactions, ${ }^{13}$ we repeated the cycle and observed a recovery of adhesion in $\mathrm{H}_{2} \mathrm{O}$ and a decline in adhesion in $\mathrm{D}_{2} \mathrm{O}$. In a separate experiment, we measured the adhesion in $\mathrm{D}_{2} \mathrm{O}$ and then in $\mathrm{H}_{2} \mathrm{O}$, and the adhesion increased correspondingly (Figure 1c). Finally, we measured the adhesion between FDTS and octadecanethiol (ODT) and observed a similar decrease in adhesion upon changing the solution from $\mathrm{H}_{2} \mathrm{O}$ to $\mathrm{D}_{2} \mathrm{O}$ and a recovery of adhesion upon changing from $\mathrm{D}_{2} \mathrm{O}$ to $\mathrm{H}_{2} \mathrm{O}$ (Figure 1d). There is a strong variation in the magnitudes of adhesion in the three experiments (Figure $1 \mathrm{~b}-\mathrm{d}$ ), which is common for hydrophobic systems and likely due to the variation of the film quality and contact mechanics. ${ }^{13}$ To minimize the effects of such variations, the contact point was always kept constant over the course of a single experiment. Over the full data set, the adhesion in $\mathrm{D}_{2} \mathrm{O}$ was $90 \pm 3 \%$ of the adhesion in $\mathrm{H}_{2} \mathrm{O}$ (Figure 1e). With the van der Waals contribution being virtually equal in $\mathrm{H}_{2} \mathrm{O}$ and $\mathrm{D}_{2} \mathrm{O}$ (see Supporting Information section I.6), the measured adhesion difference indicates that the hydrophobic force is correspondingly $\sim 10 \%$ stronger in $\mathrm{H}_{2} \mathrm{O}$ than in $\mathrm{D}_{2} \mathrm{O}$.

To gain molecular insights into our experimental observations, we performed large-scale molecular dynamics (MD) simulations using the flexible TIP4P-2005 water model. ${ }^{30}$ This model satisfactorily reproduces the properties of the bulk liquid and reasonably approximates the competing NQEs at room temperature ${ }^{31}$ (Table S2). We equilibrated the model systems comprising parallel graphene sheets and confined $\mathrm{H}_{2} \mathrm{O}$ or $\mathrm{D}_{2} \mathrm{O}$ under ambient conditions (Figure 2a, Methods, Molecular Dynamics Details), and we approximated the quantum thermodynamics [free energy, entropy, and zeropoint energy (ZPE)-corrected enthalpy] by applying the twophase thermodynamics method to classical MD trajectories ${ }^{32}$ (Figure 2a, Methods, 2PT Method Details).

Our MD simulations reveal nonlinear differences in the relative quantum free energies of interfacial $\mathrm{D}_{2} \mathrm{O}$ and $\mathrm{H}_{2} \mathrm{O}$ molecules under hydrophobic nanoconfinement compared to those in bulk $\mathrm{D}_{2} \mathrm{O}$ and $\mathrm{H}_{2} \mathrm{O}$ liquids. Figure $2 \mathrm{~b}$ presents the function for the excess density of states $\left(\mathrm{DoS}^{\mathrm{ex}}\right)$ for various separation distances $(D)$. In the instructive special case in which $D=0.7 \mathrm{~nm}$ (with a water monolayer between the graphene sheets), we find the characteristic spectral features for "free" OH ( 3660 cm ${ }^{-1}$ with this water model) and "free" OD $\left(\sim 2640 \mathrm{~cm}^{-1}\right)$. We calculate two competing thermodynamic effects due to the shifts in the vibrational properties of interfacial $\mathrm{H}_{2} \mathrm{O}$ and $\mathrm{D}_{2} \mathrm{O}$ molecules. First, the increased population of broken interfacial $\mathrm{H}$-bonding states leads to a positive ZPE correction and enthalpic destabilization (Figure $2 c$ ). Concomitantly, the intensities of the symmetric and 


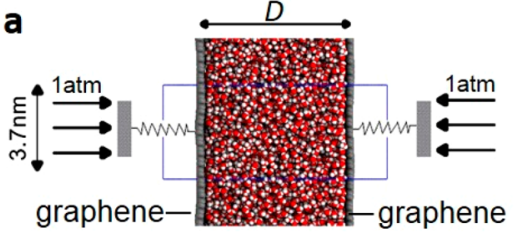

b Density of States Function
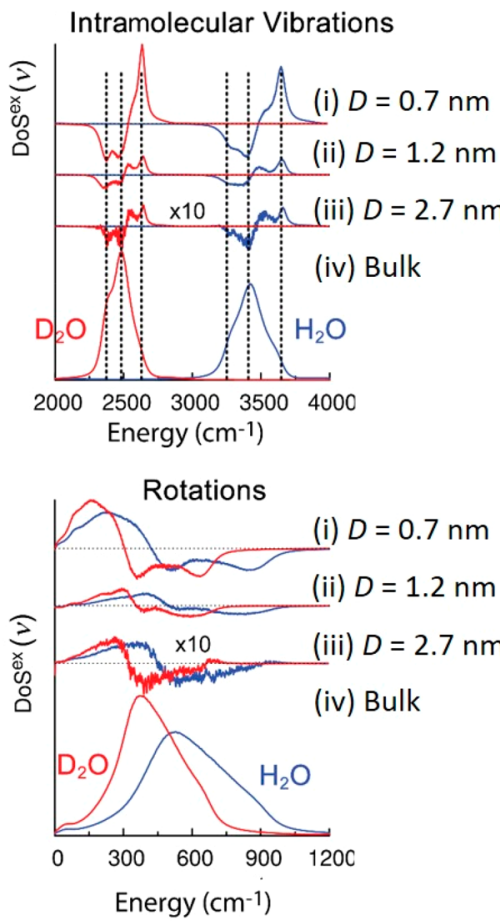

c Thermodynamics
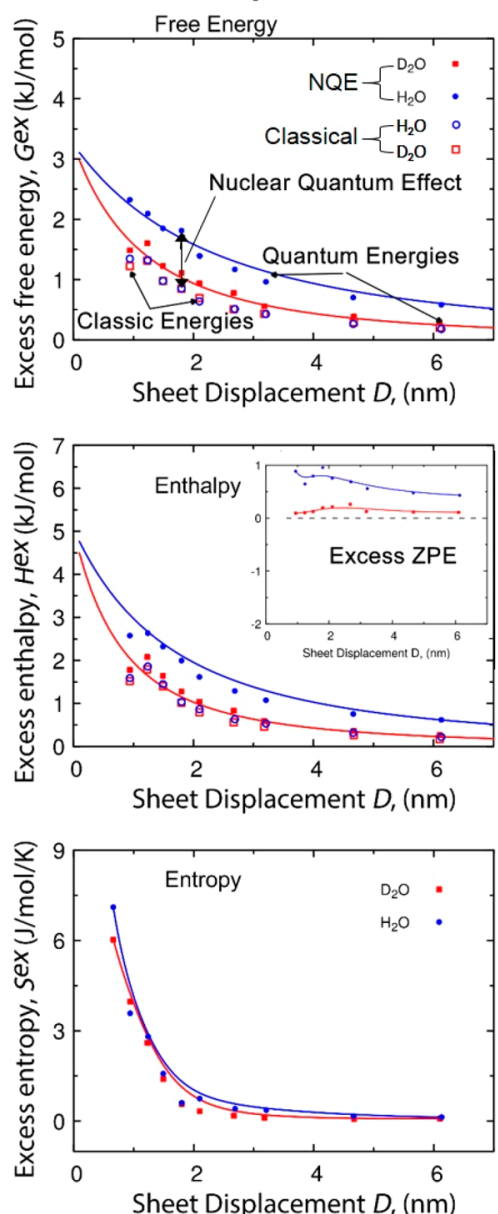

d

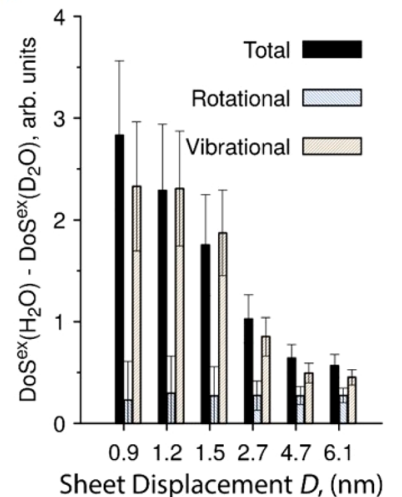

Figure 2. Thermodynamics and spectroscopy of hydrophobic nanoconfinement. (a) Schematic of our MD simulation cell. We apply 1 atm of constant pressure to the graphene sheets and vary the number of water molecules (red and white dots) between the sheets. (b) The normalized excess density of states $\left[\operatorname{DoS}^{\text {ex }}(v)\right]$ for $\mathrm{D}_{2} \mathrm{O}$ (red) and $\mathrm{H}_{2} \mathrm{O}$ (blue) with frequency $v$. Contributions arising from internal vibrations (bond stretching and angle bending, top panel) and rotations about the center of mass (bottom panel) are shown. We separately plot the $\mathrm{DoS} S^{\mathrm{ex}}(v)$ for (i) $D=0.7$ $\mathrm{nm}$, (ii) $D=1.2 \mathrm{~nm}$, and (iii) $D=2.7 \mathrm{~nm}$ sheet separations, compared to the full bulk liquid spectrum (iv). We scaled the $D=2.7 \mathrm{~nm}$ spectrum by a factor of 10 for the sake of clarity. For the internal vibrations, the dashed vertical lines indicate (with increasing frequency) the $\mathrm{O}-\mathrm{H}$ (D) symmetric and asymmetric stretches and the broken hydrogen bond peak. Convergence to the bulk DoS is observed around $D=2.7 \mathrm{~nm}$. (c) Excess per-molecule free energy (top), enthalpy (middle), and entropy (bottom) of confined $\mathrm{D}_{2} \mathrm{O}$ (red) and $\mathrm{H}_{2} \mathrm{O}$ (blue) as a function of an increase in $D$. Filled symbols represent the quantum-corrected free energies and/or enthalpies, which display a pronounced NQE between the isotopologues. Empty symbols represent the free energies and/or enthalpies obtained from the classic energies (potential energies from the MD simulations), which do not display NQEs. The middle inset shows excess zero-point energy contributions to the quantum enthalpy. (d) Plot of the difference in the excess DoS in $\mathrm{H}_{2} \mathrm{O}$ and $\mathrm{D}_{2} \mathrm{O}$ as a function of $D$. The total (black) is positive, meaning that $\mathrm{H}_{2} \mathrm{O}$ has quantum corrections that are larger than those of $\mathrm{D}_{2} \mathrm{O}$. Contributions arising from vibrational motion (gray) dominate those arising from rotational motion (pale blue).

asymmetric $\mathrm{O}-\mathrm{H}$ (D) bond stretching peaks are reduced compared to those of the bulk liquid, leading to a negative ZPE correction. Overall, the destabilizing broken $\mathrm{H}$-bonding effect dominates, and critically, $\mathrm{H}_{2} \mathrm{O}$ molecules in the first layer next to the hydrophobic interfaces are more destabilized than the $\mathrm{D}_{2} \mathrm{O}$ molecules due to the higher frequency of the $\mathrm{O}-\mathrm{H}$ bond than the $\mathrm{O}-\mathrm{D}$ bond. ${ }^{7}$ This destabilization effect provides the likely nanoscopic mechanism for the experimentally measured stronger adhesion in $\mathrm{H}_{2} \mathrm{O}$ than in $\mathrm{D}_{2} \mathrm{O}$. We also note that as previously demonstrated, ${ }^{33}$ the quasi-harmonic approximation may overestimate the nuclear quantum effect. The $10 \%$ difference measured in our SFA experiments can thus serve as a benchmark for future studies aimed at accessing the accuracy of theoretical methods of evaluating NQEs in condensed phase systems.
In addition to the shifts in the stretching properties of interfacial water molecules, we find increases in the low-energy rotational states at the interface compared to in the bulk liquid and the appearance of new peaks in the ranges of 320-450 $\mathrm{cm}^{-1}$ and $150-300 \mathrm{~cm}^{-1}$ in the spectra of interfacial $\mathrm{H}_{2} \mathrm{O}$ and $\mathrm{D}_{2} \mathrm{O}$, respectively (Figure $2 \mathrm{~b}$ ). Thus, interfacial water molecules near the hydrophobic surface have more sluggish (lower-frequency) rotational dynamics relative to the bulk liquid, ${ }^{8}$ causing an increase in the relative entropy, heat capacity, and overall stability of $\mathrm{D}_{2} \mathrm{O}$ molecules compared to those in $\mathrm{H}_{2} \mathrm{O}$, a finding recently reported by Park and coworkers from their investigation of friction at the mica-watergraphene interface. ${ }^{34}$ Finally, we find that the diffusional and librational (molecular rattling motions such as those that occur in a solid) modes are affected by the hydrophobic surface, although these effects compensate for each other and thus 
there is no appreciable difference between $\mathrm{H}_{2} \mathrm{O}$ and $\mathrm{D}_{2} \mathrm{O}$ (Figure S8).

Analysis of the stability of water molecules hydrophobically confined at larger sheet separations from our simulations shows that isotopologue NQEs extend up to the third interfacial layer, preferentially stabilizing $\mathrm{D}_{2} \mathrm{O}$ molecules approximately $\sim 1.5 \mathrm{~nm}$ from the hydrophobic surface (Figure $2 \mathrm{~d})$. Beyond these three interfacial layers, the excess free energy of $\mathrm{D}_{2} \mathrm{O}$ converges faster to the bulk $\mathrm{H}_{2} \mathrm{O}$, so that at large surface separations, the solid/liquid surface tension in $\mathrm{H}_{2} \mathrm{O}$ is similar to that in $\mathrm{D}_{2} \mathrm{O}$. We applied Young's equation ${ }^{35}$ to the calculated surface energies and found contact angles of $109^{\circ}$ and $107^{\circ}$ for $\mathrm{H}_{2} \mathrm{O}$ and $\mathrm{D}_{2} \mathrm{O}$, respectively, in good agreement with our experimental measurements. Our calculations therefore present a thermodynamic rationale for the experimental measurements. $\mathrm{H}_{2} \mathrm{O}$ molecules are relatively destabilized compared to $\mathrm{D}_{2} \mathrm{O}$ molecules due to NQEs, resulting in the larger hydrophobic force measured experimentally. This means that NQE in liquid water next to hydrophobic surfaces is a purely nanoscale phenomenon, due to the internal vibrational and rotational properties of the molecule. We confirmed this hypothesis by means of quantum $\mathrm{MD}$ simulations employing the coarse-grained $\mathrm{mW}$ water model $^{36}$ (a structureless, many-body potential), which did not show isotopologue NQEs (Figure S9a). Moreover, additional simulations uncovered the signature of NQEs on surface forces in two other nanoconfined, polar organic liquids: methanol and formic acid (Figure S9b,c).

In summary, we have advanced a thermodynamic rationale for the measured isotopic effect of hydrophobically confined water, based on considerations of NQEs arising from shifts in the rotations and intramolecular vibrations at the interface. Our findings highlight that the inclusion of NQEs in molecular simulations should yield a better understanding of intermolecular and surface phenomena in nanofluidics, ${ }^{20,37-39}$ aquatic chemistry, ${ }^{40,41,42}$ and biology. ${ }^{5}$ The preferential stabilization of $\mathrm{D}_{2} \mathrm{O}$ in hydrophobic nanoconfinement over $\mathrm{H}_{2} \mathrm{O}$ also presents advancement opportunities for separation processes at the nanoscale and nanosensor arrays with extreme sensitivity to isotopologues.

\section{METHODS}

Experimental Section. We employed a surface force apparatus (SFA) to quantify the hydrophobic interactions between $\mathrm{H}_{2} \mathrm{O}$ and $\mathrm{D}_{2} \mathrm{O}$; details of this technique have been extensively reviewed in the past. ${ }^{28}$ Briefly, the SFA uses molecularly smooth back-silvered (silver thickness of $\sim 50 \mathrm{~nm}$ to render them partially transmitting) mica surfaces of equal thickness glued onto two macroscopic cylindrical silica discs arranged in a cross-cylindrical geometry (Figure 1a). When white light passes through opposing back-silvered mica surfaces, an optical interferometer is formed. The transmission function of this interferometer is represented by a series of sharp fringes of equal chromatic order (FECO), which contain information about the thickness and the refractive indices of all of the optical layers. The distance $D$ between the surfaces is measured by white light interferometry [fringes of equal chromatic order (FECO) $]$ with an accuracy of $\pm 1 \AA^{43}$

Back-silvered mica surfaces were rendered hydrophobic by covalently depositing FDTS (see the Supporting Information for details). We brought FDTS-coated mica surfaces into contact in dry nitrogen, which defines the reference zero distance $(D=0)$. Several consecutive measurements were recorded in Milli-Q $\mathrm{H}_{2} \mathrm{O}$ (resistivity of $18 \mathrm{M} \Omega / \mathrm{cm}, \mathrm{pH} 5.6$ ), followed by $\mathrm{D}_{2} \mathrm{O}$ or vice versa, and the cycle was repeated. $\mathrm{H}_{2} \mathrm{O}$ and $\mathrm{D}_{2} \mathrm{O}$ contained $5 \mathrm{mM}$ potassium chloride (Fisher Scientific, 99.8\%) and were degassed by being stirred with Teflon-coated magnetic bars for $2 \mathrm{~h}$ before being injected into the SFA chamber. FECO images were recorded for each force run using the Andor Solis software.

Computational Details. Molecular Dynamics Details. We created model systems comprising two $2 \mathrm{D}$ periodic graphene sheets $(37 \AA \times 37 \AA)$ and adjusted the internal cavity spacing to accommodate varying numbers of water molecules (integer water layers), previously optimized from a Monte Carlo procedure, ${ }^{44,45}$ ensuring the minimum potential energy for each layer (Table S3) and assuming a van der Waals radius of $3.2 \AA$. By varying the sheet separation, we simulated the SFA experiments and tested the stability of individual water layers on the hydrophobic surfaces sequentially.

On each initial system, we then performed two-dimensional (2D) periodic ( $x$ and $y$ dimensions) equilibrium MD simulations using the LAMMPS MD simulation engine, ${ }^{46}$ with an additional force of $\pm 0.022 \mathrm{kcal} \mathrm{mol}^{-1} \AA^{-1}$ added to the top and bottom sheets, respectively, to simulate 1 atm of external pressure (Figure 2a). To remove spurious interactions between the two surfaces, we employed the $2 \mathrm{D}$ slab corrections of Yeh and Berkowitz ${ }^{47}$ with a further $3.0 z$-factor. The graphene sheets were described with the QMFF-Cx force field, ${ }^{48}$ while the water molecules were described using the TIP4P2005f $\mathrm{f}^{30}$ force field. The water-carbon van der Waals interactions were determined using the optimized parameter set from Werder et al. ${ }^{49}$ Further details about the actual MD procedure can be found in Supporting Information section II(A).

2PT Method Details. We employed an external code that implemented the 2PT method to calculate the thermodynamics from the $\mathrm{MD}$ trajectories. We approximated the quantum thermodynamic corrections to the enthalpy from our classical MD trajectories by applying quantum-corrected weighting functions to the vibrational density of states function $^{50}[\operatorname{DoS}(v)]$ at room temperature. These quantum corrections vanish at zero frequency (purely diffusive modes) and increase with an increase in frequency ${ }^{50}$ and are therefore more important for high-energy vibrations. More details about the $2 \mathrm{PT}$ method can be found in Supporting Information section $\mathrm{II}(\mathrm{B})$.

\section{ASSOCIATED CONTENT}

\section{S Supporting Information}

The Supporting Information is available free of charge on the ACS Publications website at DOI: 10.1021/acs.jpclett.9b01835.

Sample preparation, characterization, properties of light and heavy water, stability of the FDTS-coated surface, measurement of hydrophobic forces, calculation of van der Waals forces in water and heavy water, thermodynamic properties of water and confined water from MD simulations, density of states and H-bonding, and NQEs in nanoconfined liquids (PDF)

\section{AUTHOR INFORMATION}

\section{Corresponding Authors}

*E-mail: himanshu.mishra@kaust.edu.sa.

*E-mail: tpascal@ucsd.edu. 


\section{ORCID}

Stephen H. Donaldson Jr.: 0000-0001-7091-0075

Tod A. Pascal: 0000-0003-2096-1143

Himanshu Mishra: 0000-0001-8759-7812

\section{Author Contributions}

${ }$ B.R.S. and S.P. contributed equally to this work.

\section{Notes}

The authors declare no competing financial interest.

All data needed to evaluate the conclusions in the paper are present in the paper and/or the Supporting Information. Additional data related to this paper may be requested from the authors.

\section{ACKNOWLEDGMENTS}

The authors thank Professor Vishwanath Dalvi and Mr. Deepak Bapat from the Institute of Chemical Technology (Mumbai, India) and Prof. Adri van Duin and Dr. Wei Zhang (The Pennsylvania State University, University Park, PA) for fruitful discussions. S.P. thanks Mr. Sankara Arunachalam (KAUST) for assistance with contact angle goniometry. H.M. and S.P. thank Mr. Kuang-Hui Li and Dr. Miaoxiang M. Chen from KAUST and Dr. Ravi Sharma (Principal, RS Science and Technology Consulting, LLC, Acton, MA) for assistance with the functionalization of mica surfaces with perfluorodecyltrichlorosilane (FDTS). The authors thank Mr. Xavier Pita (Scientific Illustrator at KAUST) for preparing Figure ${ }^{1,} a$ and Dr. Michael Cusack and Dr. Virginia Unkefer (KAUST) for assistance in scientific editing. T.A.P. and A.S. acknowledge the Supercomputing Laboratory at KAUST in Thuwal, Saudi Arabia. Parts of the computer simulations in this work were performed as a user project at the Molecular Foundry, Lawrence Berkeley National Laboratory, supported by the Office of Science, Office of Basic Energy Sciences, of the U.S. Department of Energy (DOE). Further computer simulations were performed at the National Energy Research Scientific Computing Center, which is supported by the Office of Science of the DOE. S.H.D. acknowledges funding support from LabEX ENS-ICFP (ANR-10-LABX-0010 and ANR-10IDEX-0001-02 PSL). H.M., T.A.P., and S.H.D. acknowledge KAUST Office of Sponsored Research Competitive Research Grant OSR-CRG2017-3415.

\section{REFERENCES}

(1) Markland, T. E.; Ceriotti, M. Nuclear quantum effects enter the mainstream. Nat. Rev. Chem. 2018, 2 (3), 1-14.

(2) Paesani, F.; Voth, G. A. The Properties of Water: Insights from Quantum Simulations. J. Phys. Chem. B 2009, 113 (17), 5702-5719.

(3) Ceriotti, M.; Fang, W.; Kusalik, P. G.; McKenzie, R. H.; Michaelides, A.; Morales, M. A.; Markland, T. E. Nuclear Quantum Effects in Water and Aqueous Systems: Experiment, Theory, and Current Challenges. Chem. Rev. 2016, 116, 7529-7550.

(4) Berger, A.; Ciardi, G.; Sidler, D.; Hamm, P.; Shalit, A. Impact of nuclear quantum effects on the structural inhomogeneity of liquid water. Proc. Natl. Acad. Sci. U. S. A. 2019, 116, 2458-2463.

(5) Pereyaslavets, L.; Kurnikov, I.; Kamath, G.; Butin, O.; Illarionov, A.; Leontyev, I.; Olevanov, M.; Levitt, M.; Kornberg, R. D.; Fain, B. On the importance of accounting for nuclear quantum effects in $a b$ initio calibrated force fields in biological simulations. Proc. Natl. Acad. Sci. U. S. A. 2018, 115, 8878-8882.

(6) Soper, A. K.; Benmore, C. J. Quantum differences between heavy and light water. Phys. Rev. Lett. 2008, 101 (6), 065502.

(7) De Marco, L.; Carpenter, W.; Liu, H.; Biswas, R.; Bowman, J. M.; Tokmakoff, A. Differences in the Vibrational Dynamics of $\mathrm{H} 2 \mathrm{O}$ and D2O: Observation of Symmetric and Antisymmetric Stretching
Vibrations in Heavy Water. J. Phys. Chem. Lett. 2016, 7 (10), 17691774.

(8) Bonn, M.; Nagata, Y.; Backus, E. H. G. Molecular Structure and Dynamics of Water at the Water-Air Interface Studied with SurfaceSpecific Vibrational Spectroscopy. Angew. Chem., Int. Ed. 2015, 54 (19), 5560-5576.

(9) Davis, J. G.; Gierszal, K. P.; Wang, P.; Ben-Amotz, D. Water structural transformation at molecular hydrophobic interfaces. Nature 2012, 491 (7425), 582-585.

(10) Lum, K.; Chandler, D.; Weeks, J. D. Hydrophobicity at Small and Large Length Scales. J. Phys. Chem. B 1999, 103, 4570-4577.

(11) Israelachvili, J.; Pashley, R. The Hydrophobic Interaction Is Long-Range, Decaying Exponentially with Distance. Nature 1982, 300 (5890), 341-342.

(12) Tabor, R. F.; Wu, C.; Grieser, F.; Dagastine, R. R.; Chan, D. Y. C. Measurement of the Hydrophobic Force in a Soft Matter System. J. Phys. Chem. Lett. 2013, 4 (22), 3872-3877.

(13) Donaldson, S. H., Jr; Røyne, A.; Kristiansen, K.; Rapp, M. V.; Das, S.; Gebbie, M. A.; Lee, D. W.; Stock, P.; Valtiner, M.; Israelachvili, J. Developing a general interaction potential for hydrophobic and hydrophilic interactions. Langmuir 2015, 31 (7), 2051-2064.

(14) Ma, C. D.; Wang, C. X.; Acevedo-Velez, C.; Gellman, S. H.; Abbott, N. L. Modulation of hydrophobic interactions by proximally immobilized ions. Nature 2015, 517 (7534), 347-U443.

(15) Ben-Naim, A. Hydrophobic Interactions; Plenum Press, 1980.

(16) Hummer, G.; Garde, S.; Garcla, A.; Pratt, L. New perspectives on hydrophobic effects. Chem. Phys. 2000, 258 (2), 349-370.

(17) Oakenfull, D.; Fenwick, D. E. Thermodynamics and mechanism of hydrophobic interaction. Aust. J. Chem. 1977, 30 (4), $741-752$.

(18) Gallo, A.; Farinha, A. S. F.; Dinis, M.; Emwas, A.-H.; Santana, A.; Nielsen, R. J.; Goddard, W. A.; Mishra, H. The chemical reactions in electrosprays of water do not always correspond to those at the pristine air-water interface. Chemical Science 2019, 10 (9), 25662577.

(19) Chen, Y. X.; Okur, H. I.; Gomopoulos, N.; Macias-Romero, C.; Cremer, P. S.; Petersen, P. B.; Tocci, G.; Wilkins, D. M.; Liang, C. W.; Ceriotti, M.; Roke, S. Electrolytes induce long-range orientational order and free energy changes in the H-bond network of bulk water. Sci. Adv. 2016, 2 (4), e1501891.

(20) Fumagalli, L.; Esfandiar, A.; Fabregas, R.; Hu, S.; Ares, P.; Janardanan, A.; Yang, Q.; Radha, B.; Taniguchi, T.; Watanabe, K.; Gomila, G.; Novoselov, K. S.; Geim, A. K. Anomalously low dielectric constant of confined water. Science 2018, 360 (6395), 1339-1342.

(21) Holt, J. K.; Park, H. G.; Wang, Y.; Stadermann, M.; Artyukhin, A. B.; Grigoropoulos, C. P.; Noy, A.; Bakajin, O. Fast Mass Transport Through Sub-2-Nanometer Carbon Nanotubes. Science 2006, 312 (5776), 1034-1037.

(22) Chandler, D. Interfaces and the driving force of hydrophobic assembly. Nature 2005, 437 (7059), 640-647.

(23) Sanchez-Iglesias, A.; Grzelczak, M.; Altantzis, T.; Goris, B.; Perez-Juste, J.; Bals, S.; Van Tendeloo, G.; Donaldson, S. H.; Chmelka, B. F.; Israelachvili, J. N.; Liz-Marzan, L. M. Hydrophobic Interactions Modulate Self-Assembly of Nanoparticles. ACS Nano 2012, 6 (12), 11059-11065.

(24) Tanford, C.; Reynolds, J. Nature's Robots: A History of Proteins; Oxford University Press: New York, 2003.

(25) Israelachvili, J. N. Intermolecular and Surface Forces: Revised Third Edition; Academic Press, 2011.

(26) Mishra, H.; Schrader, A. M.; Lee, D. W.; Gallo, A.; Chen, S. Y.; Kaufman, Y.; Das, S.; Israelachvili, J. N. Time-Dependent Wetting Behavior of PDMS Surfaces with Bioinspired, Hierarchical Structures. ACS Appl. Mater. Interfaces 2016, 8 (12), 8168-8174.

(27) Li, Z. T.; Wang, Y. J.; Kozbial, A.; Shenoy, G.; Zhou, F.; McGinley, R.; Ireland, P.; Morganstein, B.; Kunkel, A.; Surwade, S. P.; Li, L.; Liu, H. T. Effect of airborne contaminants on the wettability of supported graphene and graphite. Nat. Mater. 2013, 12 (10), 925931. 
(28) Israelachvili, J.; Min, Y.; Akbulut, M.; Alig, A.; Carver, G.; Greene, W.; Kristiansen, K.; Meyer, E.; Pesika, N.; Rosenberg, K.; Zeng, H. Recent advances in the surface forces apparatus (SFA) technique. Rep. Prog. Phys. 2010, 73 (3), 036601.

(29) Cui, X.; Liu, J.; Xie, L.; Huang, J.; Liu, Q.; Israelachvili, J. N.; Zeng, H. Modulation of Hydrophobic Interaction by Mediating Surface Nanoscale Structure and Chemistry, not Monotonically by Hydrophobicity. Angew. Chem., Int. Ed. 2018, 57 (37), 11903-11908.

(30) González, M. A.; Abascal, J. L. A flexible model for water based on TIP4P/2005. J. Chem. Phys. 2011, 135 (22), 224516.

(31) Habershon, S.; Markland, T. E.; Manolopoulos, D. E. Competing quantum effects in the dynamics of a flexible water model. J. Chem. Phys. 2009, 131 (2), 024501.

(32) Lin, S.-T.; Maiti, P. K.; Goddard, W. A., III Two-phase thermodynamic model for efficient and accurate absolute entropy of water from molecular dynamics simulations. J. Phys. Chem. B 2010, 114 (24), 8191-8198.

(33) Ceriotti, M.; Markland, T. E. Efficient methods and practical guidelines for simulating isotope effects. J. Chem. Phys. 2013, 138 (1), 014112 .

(34) Lee, H.; Ko, J.-H.; Song, H. C.; Salmeron, M.; Kim, Y.-H.; Park, J. Y. Isotope- and Thickness-Dependent Friction of Water Layers Intercalated Between Graphene and Mica. Tribol. Lett. 2018, 66 (1), 36.

(35) Kaufman, Y.; Chen, S.-Y.; Mishra, H.; Schrader, A. M.; Lee, D. W.; Das, S.; Donaldson, S. H.; Israelachvili, J. N. Simple-to-Apply Wetting Model to Predict Thermodynamically Stable and Metastable Contact Angles on Textured/Rough/Patterned Surfaces. J. Phys. Chem. C 2017, 121 (10), 5642-5656.

(36) Molinero, V.; Moore, E. B. Water modeled as an intermediate element between carbon and silicon. J. Phys. Chem. B 2009, 113 (13), 4008-4016.

(37) Pascal, T. A.; Goddard, W. A.; Jung, Y. Entropy and the driving force for the filling of carbon nanotubes with water. Proceedings of the National Academy of Sciences 2011, 108 (29), 11794.

(38) Das, R.; Arunachalam, S.; Ahmad, Z.; Manalastas, E.; Mishra, $\mathrm{H}$. Bio-inspired gas-entrapping membranes (GEMs) derived from common water-wet materials for green desalination. Journal of Membrane Science 2019, 588, 117185.

(39) Subramanian, N.; Qamar, A.; Alsaadi, A.; Gallo, A.; Ridwan, M. G.; Lee, J.-G.; Pillai, S.; Arunachalam, S.; Anjum, D.; Sharipov, F.; Ghaffour, N.; Mishra, H. Evaluating the potential of superhydrophobic nanoporous alumina membranes for direct contact membrane distillation. Journal of Colloid and Interface Science 2019, 533, 723-732.

(40) Mishra, H.; Enami, S.; Nielsen, R. J.; Stewart, L. A.; Hoffmann, M. R.; Goddard, W. A.; Colussi, A. J. Bronsted basicity of the airwater interfac. P Natl Acad Sci USA 2012, 109 (46), 18679-18683.

(41) Mishra, H.; Enami, S.; Nielsen, R. J.; Hoffmann, M. R.; Goddard, W. A.; Colussi, A. J. Anions dramatically enhance proton transfer through aqueous interfaces. P Natl Acad Sci USA 2012, 109 (26), 10228-10232.

(42) Gallo, A.; Farinha, A. S. F.; Emwas, A.-H.; Santana, A.; Nielsen, R. J.; Goddard, W. A.; Mishra, H. Reply to the Comment on "The chemical reactions in electrosprays of water do not always correspond to those at the pristine air-water interface". A. J. Colussi and S. Emani, Chem. Sci. 2019, 10, DOI: 10.1039/c9sc00991d.

(43) Israelachvili, J. N. Thin film studies using multiple-beam interferometry. J. Colloid Interface Sci. 1973, 44 (2), 259-272.

(44) Maiti, P. K.; Çağın, T.; Wang, G.; Goddard, W. A. Structure of PAMAM dendrimers: Generations 1 through 11. Macromolecules 2004, 37 (16), 6236-6254.

(45) Cagin, T.; Wang, G.; Martin, R.; Breen, N.; Goddard, W. A., III Molecular modelling of dendrimers for nanoscale applications. Nanotechnology 2000, 11 (2), 77.

(46) Plimpton, S. Fast parallel algorithms for short-range molecular dynamics. J. Comput. Phys. 1995, 117 (1), 1-19.

(47) Yeh, I.-C.; Berkowitz, M. L. Ewald summation for systems with slab geometry. J. Chem. Phys. 1999, 111 (7), 3155-3162.
(48) Pascal, T. A.; Karasawa, N.; Goddard, W. A., III Quantum mechanics based force field for carbon (QMFF-Cx) validated to reproduce the mechanical and thermodynamics properties of graphite. J. Chem. Phys. 2010, 133 (13), 134114.

(49) Werder, T.; Walther, J. H.; Jaffe, R.; Halicioglu, T.; Koumoutsakos, P. On the water- carbon interaction for use in molecular dynamics simulations of graphite and carbon nanotubes. $J$. Phys. Chem. B 2003, 107 (6), 1345-1352.

(50) Berens, P. H.; Mackay, D. H.; White, G. M.; Wilson, K. R. Thermodynamics and quantum corrections from molecular dynamics for liquid water. J. Chem. Phys. 1983, 79 (5), 2375-2389. 